\title{
El papel del Estado y los paradigmas económicos en América Latina
}

\author{
Enrique V. Iglesias
}

$\mathrm{E}$

ste artículo examina los dos paradigmas de desarrollo que sucesivamente prevalecieron en América Latina en la segunda mitad del siglo XX, concentrándose en el papel asignado al Estado. En el paradigma cepalino se ve cómo amplias tareas de guía, estimulador y partícipe directo en el desarrollo productivo fueron perdiendo eficacia, a medida que el Estado era capturado por intereses particulares sin que instituciones sólidas fueran capaces de ponerle coto. Posteriormente, la extrema debilitación del Estado experimentada desde la vigencia del Consenso de Washington impidió la adecuada regulación de las actividades privatizadas e hizo perder la visión de largo plazo y la preocupación por la concentración de ingresos. El artículo alude a la demanda social por un nuevo tipo de Estado y analiza los requisitos y objetivos para que, entre otras cosas, este facilite un funcionamiento eficaz de los mercados y se preocupe por disminuir las desigualdades

Secretaría General Iberoamericana (SEGIB)

Ex Presidente del Banco Interamericano

de Desarrollo (BID)

Ex Secretario Ejecutivo de la CEPAL

• eiglesias@segib.org sociales. 


\section{I}

\section{Introducción}

Por una amable invitación que me extendiera mi gran amigo y colega José Luis Machinea, participé recientemente en una reunión muy especial de la CEPAL, convocada para conmemorar a Raúl Prebisch, insigne economista de nuestra región y contemporáneo ilustre de muchos de los que se hallaban en ese acto. Su memoria nos evoca la época de oro del pensamiento económico del desarrollo en la segunda mitad del siglo pasado, que él lideró con tanta sabiduría en nuestra América Latina y que proyectó al ámbito académico internacional y de prestigiosas instituciones especializadas, como la Comisión Económica para América Latina y el Caribe, el Instituto Latinoamericano y del Caribe de Planificación Económica y Social, y la Conferencia de las Naciones Unidas sobre Comercio y Desarrollo. Su vida fue rica en pensamiento y acción, que en el transcurso del tiempo ha inspirado la formación de nuevas generaciones de profesionales comprometidos con la causa del progreso económico y social de nuestros pueblos, a la vez que sus ideas son valoradas con debida justicia por numerosos y destacados economistas del mundo académico actual. Para mí su memoria es verdaderamente emocionante, como el maestro que abrió mis ojos al mundo de la economía real latinoamericana y mundial y como el amigo generoso cuyos ejemplos, consejos y apoyo fueron determinantes en mi carrera y dedicación al desarrollo económico y social de América Latina y el Caribe. A ello se unió en ese momento mi nostalgia de los años pasados en la que fue y siento mi casa, la CEPAL, cuna de ensueños y experiencias compartidas a través de tantos años. Mucho agradezco la oportunidad que se me brindó para sentir el reencuentro con las memorias que ella encierra y con tan buenos amigos y compañeros de ruta.

En esa ocasión compartí, con quienes nos acompañaban, algunas reflexiones sobre el papel del Estado en los escenarios orientados por la búsqueda de paradigmas económicos en nuestra región, ya por más de medio siglo. En este artículo hago lo mismo. No pretendo enunciar un ensayo teórico, sino más bien ilustrar la visión de un practicante privilegiado en las tareas de la disquisición académica y en la praxis de las políticas de desarrollo de la gran mayoría de los países en la región.

\section{II}

\section{Los paradigmas económicos}

La búsqueda de paradigmas económicos ha sido una constante en la región desde mediados del siglo pasado. Pocas regiones han conocido un debate tan encendido y ensayos tan variados como América Latina. Nuestra región ha constituido un verdadero laboratorio de ideas y propuestas de política impulsadas por las ideologías de turno, en muchos casos siguiendo enseñanzas originadas en otras partes del mundo.

De esa búsqueda se derivan lecciones diversas. Una primera lección aprendida en mi experiencia de casi medio siglo es que el subdesarrollo económico es mucho más complejo que lo que pensábamos hace cincuenta años.

$\square$ Este artículo recoge la conferencia magistral dictada por el autor en la Comisión Económica para América Latina y el Caribe (Santiago de Chile, 28 de agosto de 2006), en el marco de la Sexta Cátedra Raúl Prebisch.
Y ello a pesar de que en aquella época se realizaron grandes esfuerzos de reflexión. La CEPAL fue, en cierta medida, un semillero de ideas impulsadas con tanto brillo por Raúl Prebisch. Pero ella fue también un centro de investigación y conocimiento de la realidad económica de cada país, en numerosos casos con la cooperación estrecha de los gobiernos. Como parte de esa vivencia, aprendimos a conocer mejor la complejidad económica de cada país y de su entorno internacional.

Así, una lección muy importante de esa experiencia de tantos años es saber evitar los reducimientos o simplificaciones excesivas de la realidad que suelen acompañar la búsqueda de los paradigmas. Esas simplificaciones han estado presentes casi en todas las formulaciones teóricas y sus respectivas propuestas de política. En mi experiencia personal he observado dos tipos de simplificaciones excesivas: las del ámbito económico y las relativas a los marcos social y político. 
¿Cómo superar las simplificaciones de la teoría? El avance del análisis macroeconómico ha hecho una enorme contribución al mejoramiento de la formulación de políticas. Los modelos macroeconómicos han progresado extraordinariamente en su capacidad de análisis y de prescripción de políticas. Tres áreas en las que ese avance ha sido notable y útil corresponden al campo monetario y financiero; al de formación de los precios, inclusive la inflación, y al de comercio exterior.

Sin embargo, me parece que en muchos casos se ha sacrificado la realidad en beneficio de la elegancia formal y matemática de los modelos. No es cuestión de menospreciar la contribución de los modelos macroeconómicos al conocimiento y la praxis de la política, pero a veces se tiende a anteponer su elegancia formal a la complejidad de la realidad económica, social y política.

Talvez, para fines ilustrativos, podamos hacer un símil con la construcción de una casa. Para esto se requiere el aporte de un buen ingeniero que asegure la idoneidad de sus cimientos e infraestructura. Simplemente no habrá casa segura sin ello. Pero, además, se requiere la contribución del arquitecto que aporte el diseño apropiado a las aspiraciones y necesidades de sus moradores. Pienso que en la búsqueda de paradigmas económicos ocurre algo semejante. Se necesitan buenos ingenieros económicos para asegurar la coherencia de los modelos económicos y formular sus condiciones de óptimo. Pero también se necesitan arquitectos económicos que aseguren la correspondencia entre la racionalidad de la ingeniería económica y las exigencias de la realidad social y política. Considero que el avance en la ingeniería económica ha superado lejos a la arquitectura de los paradigmas económicos. Las experiencias de los años pasados muestran a menudo que ha habido déficit en cuanto al diseño de la casa, mientras abundó la elegancia formal de su estructura.

¿Cuál ha sido la experiencia con relación a los paradigmas sociopolíticos? En los empeños por conocer y aprehender la realidad se ha recurrido a ideas y categorías de análisis sociológico y político. Por ahí nos acercamos a una visión integral de la realidad. Sin embargo, esa comprensión de los factores sociales o políticos condicionantes ha sido en muchos casos seducida inevitablemente por ideologías o concepciones apriorísticas, que siendo útiles para entender los fenómenos sociales, corren el riesgo de colocar las ideologías delante de las realidades. Y esto conlleva una simplificación tan peligrosa como la anterior.

Por lo tanto, una de las lecciones más valiosas de los años pasados es la necesidad de evitar uno u otro tipo de simplificación. En un caso arriesgamos el quedarnos con economías sin sociedad. $\mathrm{Y}$ en el otro, quedarnos con sociedad sin economía. Los paradigmas encierran uno u otro riesgo, por lo que debemos cuidarnos de las simplificaciones excesivas si queremos tener una visión integral de la realidad sobre la cual aplicar políticas económicas y sociales relevantes y viables.

Lo anterior no significa ignorar el papel de las ideas y de las ideologías, que han probado ser tan valiosas a lo largo de la historia. Lo que reconozco en mi experiencia personal es que en la búsqueda de los paradigmas económicos y sociales es preciso aprehender las realidades nacionales e internacionales, y en ellas identificar los obstáculos y condiciones impuestos por la aplicación de las distintas propuestas de política.

Entre los factores condicionantes de las realidades económicas, políticas y sociales, las instituciones tienen un papel crucial, como se ha venido reconociendo cada vez más. Y entre ellas se destaca el Estado. En América Latina un factor fundamental de los grandes paradigmas de desarrollo ha sido el papel asignado al Estado.

Este tema no es nuevo, pero me propongo abordarlo aquí porque puede ser de interés mirarlo a la luz de mi experiencia personal, aprovechando especialmente los resultados de los trabajos que realizamos en el Banco Interamericano de Desarrollo (BID) en años recientes. Allí montamos una unidad dedicada especialmente al análisis de los problemas políticos en el desarrollo latinoamericano y, en particular, del papel del Estado. Con ello simplemente nos sumamos a la corriente institucionalista de los últimos años, que ha venido poniendo de relieve el rol fundamental de las instituciones en lo que se refiere a explicar y combatir los problemas del desarrollo.

Quisiera discurrir aquí sobre el papel del Estado en cada uno de los dos principales paradigmas de política económica de los últimos 50 años en América Latina: el paradigma de la CEPAL y el del Consenso de Washington. De ese examen y de las lecciones aprendidas surge la necesidad de repensar el rol del Estado en el marco de las nuevas realidades de América Latina y de las estrategias económicas que se están aplicando en la región.

\section{El paradigma cepalino}

El primer gran encuentro entre Estado y paradigma que nuestra generación observó en América Latina surgió de la propuesta cepalina. Como bien sabemos, el marco de este paradigma incluyó un conjunto de ideas fuerza, entre las que se destacan: i) el encuadre del paradigma en la relación centro-periferia; ii) la orientación del desarrollo hacia adentro; iii) el papel de la tecnología; 
iv) la industrialización sustitutiva, y v) el rol activo del Estado. A su vez, la implementación de esa estrategia de desarrollo descansaba en un conjunto de instrumentos y políticas específicas de carácter arancelario, tributario, cambiario, crediticio y de incentivos fiscales al desarrollo industrial, y en la atención del crecimiento explosivo de las demandas sociales generadas por las migraciones del campo a las ciudades.

En ese contexto correspondía al Estado cumplir un papel protagónico, para lo cual se crearon ministerios especializados, oficinas de planificación, y bancos de desarrollo destinados a movilizar recursos financieros y tecnologías. La ampliación y fortalecimiento del aparato estatal fue el instrumento básico de la política económica. El desarrollo impulsado por esta estrategia transformó profundamente el perfil económico y social latinoamericano. Un aspecto destacado de la transformación social lo constituyó el intenso proceso de urbanización. En el plano económico, el sector manufacturero aumentó su gravitación en el producto global y en el empleo, mientras declinaba o se estancaba la producción agrícola, se aceleraba la expansión de los servicios e inclusive el aumento del empleo en el sector público, y adquirían una ponderación cada vez mayor las burocracias y la absorción de recursos por parte del Estado. La población urbana alcanzó niveles de ingreso crecientes y sensiblemente más altos que los del ámbito rural, aunque su dinámica expansiva se debilitó marcadamente entre la década de 1960 y la de 1970.

¿Por qué ese agotamiento progresivo del desarrollo latinoamericano? ¿No fueron esos mismos postulados de las políticas de desarrollo los aplicados exitosamente por los "tigres asiáticos"? ¿Por qué entonces América Latina no consiguió hacer viable una economía industrial progresiva y, en cambio, perdió posiciones significativas frente a países que iniciaron su industrialización desde bases más débiles? Ciertamente las causas fueron muchas, pero hubo factores que tuvieron una incidencia nefasta importante en los resultados.

El Estado asiático disfrutó de mayor autonomía que el latinoamericano, y estuvo precedido por tradiciones burocráticas eficientes y libres de la influencia de intereses particulares. En cambio, la experiencia en América Latina fue muy distinta, no tanto por la naturaleza de las políticas, sino más bien por su implementación. Las bases fiscales fueron insuficientes, debido en gran medida a la ausencia de un auténtico pacto social y político redistributivo. El Estado fue facilmente dominado por intereses particulares, entre otros, los de las agrupaciones o partidos políticos, grupos económicos, líderes militares, caudillos o dictadores, los cuales fortalecieron su poder político y económico por la vía del Estado. Eso explica también el carácter clientelista del empleo y de la gestión del gasto público por los regímenes autoritarios o semidemocráticos.

En suma, el Estado que acompañó al paradigma cepalino fue omnipresente, centralista y cautivo. Además, en pocos países de América Latina se consiguió avanzar en la construcción de un sólido Estado democrático, condición indispensable para asegurar la autonomía frente a los intereses particulares y la confianza en el imperio de la ley. Estas deficiencias condujeron a la inestabilidad política y al déficit democrático.

En los estudios hechos en el BID se analizó ese déficit democrático a partir de dos relaciones: las del Estado con el mercado y las del Estado con la sociedad. Los vacíos o fallas en esas relaciones erosionaron la viabilidad de un desarrollo sustentable y equitativo.

La usurpación del Estado por los intereses privados condujo a intervenciones estatales que entorpecieron el funcionamiento eficiente del mercado y promovieron el rentalismo, la especulación y la corrupción. A su vez, las políticas públicas capturadas por intereses particulares no pudieron responder a las demandas de la mayoría de los ciudadanos, contribuyendo así a excluir de los beneficios del crecimiento a amplios sectores de la población y a la pérdida de legitimidad del Estado.

El agotamiento del modelo heterodoxo de la CEPAL precipitó la crisis del Estado. No es esta la oportunidad de examinar las causas del agotamiento de ese modelo. Lo cierto es que la crisis sufrida en la década de 1980, que tuvo su máxima expresión en la crisis de la deuda, aceleró los procesos inflacionarios, acentuó la pérdida de competitividad de la economía y aumentó las distancias sociales. En el plano institucional, se destruyeron los bancos centrales, los ministerios de planificación y las instituciones financieras y de fomento. Pero igualmente grave, o aun peor, fue que en la formulación de la política de desarrollo se perdieron las perspectivas de largo plazo. Los problemas de la sobrevivencia económica y las crisis de corto plazo acapararon la atención de las políticas en la mayoría de los países. Esto y, en especial, los problemas derivados de la crisis de la deuda, nos llamaron a principios del decenio de 1980 a alertar desde la CEPAL sobre la inminencia de una "década perdida", la que de hecho y lamentablemente ocurrió y cuyos graves efectos adversos se proyectaron a los ámbitos económico y social de nuestros países.

La gravedad de la crisis llevó al abandono del modelo heterodoxo y al retorno del modelo ortodoxo y la adopción del Consenso de Washington. 


\section{El Consenso de Washington}

El retorno de la ortodoxia significó la adopción de las reglas de juego del mercado, la vigencia del sistema de precios como principal mecanismo de asignación de recursos, la aplicación de fuertes programas de estabilización, la apertura al comercio internacional, el ingreso de recursos financieros y de la inversión privada extranjera, y una política generalizada de privatizaciones. Las reformas económicas fueron alentadas en buena medida por los organismos financieros en Washington, en particular los surgidos de la Conferencia de Bretton Woods. Estos constituyeron un instrumento importante para impulsar la realización de las reformas.

La concepción del Estado liberal patrocinado por el Consenso de Washington estuvo dominada por una actitud antiestatista generalizada, que basó su justificación en la crisis enfrentada por el Estado heterodoxo, así como en su ineficiencia, el peso de la burocracia y especialmente la corrupción.

Asimismo, no dejó de tener influencia la expansión generalizada del mercado en un creciente número de economías emergentes, tanto entre los países en desarrollo como en la esfera socialista.

El nuevo concepto de Estado lo caracterizó como minimalista y prescindente. Se estimó que abundaban los argumentos para descalificar al Estado como mecanismo de asignación de recursos, principalmente los relativos a su ineficiencia, la corrupción, el clientelismo y su desborde burocrático. Esto llevó a patrocinar un Estado minimizado, lo cual significó el cierre de instituciones, la eliminación de instrumentos de política y el recorte de su intervencionismo excesivo. Por ejemplo, las políticas industriales y agrícolas fueron eliminadas. Y, sobre todo, se perdió la visión de largo plazo.

Así, las reformas cayeron en un grave error, el de dar la espalda al Estado. La prescindencia del Estado en la realización de las reformas hizo que estas experimentaran una pérdida generalizada de credibilidad. Según las encuestas de Latinobarómetro, solamente un tercio de la población latinoamericana cree en las reformas. Esto condujo a una creciente pérdida de legitimidad de las reformas y del mercado en las grandes mayorías de la población. Esa crisis de credibilidad se sumó al sentimiento generalizado de frustración y de fatiga debido a la insuficiencia del progreso y la dureza de los sacrificios hechos en la aplicación de las reformas.

Ahora bien, ¿cómo se relaciona el Estado con el mercado y con los ciudadanos en este nuevo paradigma?

En cuanto a su relación con el mercado, hubo cambios importantes en las políticas económicas, como: i) las privatizaciones y la apertura al mercado de sectores completos de la economía; ii) el debilitamiento o la ausencia de los marcos de regulación requeridos para fomentar la competencia y proteger el interés de los consumidores; iii) la oposición continuada de los intereses corporativos a los procesos de reforma; iv) la falta de mecanismos adecuados de concertación entre ganadores y perdedores de la aplicación de las reformas; v) el abandono de las medidas de promoción de sectores productivos específicos; vi) el escaso avance en la realización de reformas fiscales auténticas, y vii) una baja y decreciente inversión pública en infraestructura.

Respecto a la relación del Estado con los ciudadanos resaltan algunos aspectos importantes. En muchos países siguen ausentes las condiciones necesarias para un auténtico imperio de la ley y la justicia. Por una parte, los sistemas judiciales son poco confiables, lo cual acentúa la falta de seguridad jurídica. A su vez, el Estado carece de la capacidad necesaria para promover pactos sociales redistributivos.

Analicemos ahora cuál ha sido la atención prestada por las nuevas estrategias del desarrollo a la definición del papel del Estado. En general, en los últimos años del siglo XX y primeros años del siglo XXI ha resurgido una marcada preocupación por el desarrollo social. De una manera u otra, también se han sentido en todo el mundo los efectos de los trágicos acontecimientos del 11 de septiembre de 2001, destacándose entre ellos el énfasis en las medidas de seguridad impulsadas por el Estado.

La relación del Estado con el mercado y los ciudadanos en este nuevo paradigma conllevó una baja capacidad de la administración pública para elaborar e implementar las políticas, debido en gran medida a las crisis fiscales sufridas. Las reformas de la administración pública derivaron más en reformas fiscales que en ejercicios específicos de reorganización. En los procesos de reforma de la administración pública se privilegió su orientación tecnocrática y se descuidó la realización de un auténtico cambio de estructura del Estado.

Por ello, en los últimos años ha surgido el interés por redefinir el papel del Estado en el contexto de las nuevas estrategias de desarrollo y, como lo señalamos anteriormente, se ha hecho hincapié en la preocupación por el desarrollo social y, desde el año 2001, en las medidas de seguridad nacional. Esto coincide con tendencias favorables en el plano económico internacional, un crecimiento significativo del producto y del comercio mundial, el mejoramiento de los precios de las materias primas y la expansión de los flujos financieros y de la inversión privada extranjera. Por 
cierto, este clima de bonanza económica internacional ha tenido efectos favorables para América Latina, a lo cual se suma la buena gestión macroeconómica que hoy prevalece en la mayoría de nuestros países. En ellos tanto la gestión de la deuda externa como el desempeño exportador han enfrentado condiciones externas favorables.

Las nuevas estrategias económicas se proyectan en un marco general en que prevalecen, en el plano externo, condiciones de balanza de pagos más favorables que en el pasado y la presencia de nuevos actores en el escenario internacional, como China e India, que ofrecen grandes oportunidades a la región y a la vez plantean grandes desafíos; y en el orden interno, la consolidación de una buena gestión macroeconómica, una renovada atención a los problemas macroeconómicos, y la revindicación tanto de las políticas públicas especialmente orientadas a corregir fallas de mercado como de la acción del Estado. En todos estos temas la CEPAL ha venido trabajando desde su misma creación.

¿Estamos acaso en presencia de un nuevo paradigma? Sinceramente no lo creo y prefiero entenderlo como un paradigma incremental. En la región hay un alto pragmatismo aprendido de las experiencias propias y ajenas, así como existe también una mayor conciencia de las limitaciones que las relaciones internacionales imponen en el mundo moderno. En el acercamiento en la relación entre el Estado y el mercado prevalece lo expresado en esta ciudad por el Presidente Ricardo Lagos: más mercado y mejor Estado. Por cierto, a cada país le corresponde definir apropiadamente las relaciones entre el mercado y el Estado.

\section{III}

\section{Un nuevo concepto del Estado: sus objetivos y los requisitos para alcanzarlos}

El tema sobre el que debemos reflexionar es, por tanto, la noción del nuevo concepto del Estado, aprovechando las buenas y malas lecciones dejadas por su papel en los dos paradigmas precedentes. A partir de esas experiencias, quisiera identificar ahora algunos objetivos que me parecen relevantes en la búsqueda de ese nuevo Estado.

\section{Objetivos}

Un primer objetivo es el de lograr un Estado capaz de hacer viable la eficiencia del mercado. El Estado es importante, si acaso no indispensable, para la consecución de un mercado eficiente. Esto supone la existencia de un sistema legal y judicial cierto y creíble en su desempeño, que garantice la efectividad de los derechos de propiedad e individuales. A ello se suma la necesidad de marcos de regulación que aseguren el equilibrio entre los intereses públicos y privados. También es necesaria la creación y defensa de una competencia que garantice la eficiencia del mercado. La experiencia nos enseña cuán nefastos son los resultados de la privatización de empresas estatales cuando, por la falta de las condiciones antes señaladas, se reemplaza el monopolio público por el privado. En suma, para que el mercado funcione con eficiencia, en términos de crecimiento y de generación de oportunidades para toda la población, se necesita un Estado capaz de intervenir eficazmente donde le corresponde, aunque no debe hacerlo donde no le corresponde.

Segundo, se necesita un Estado impulsor de la capacidad productiva, con lo cual no abogamos por una propuesta apriorística de un Estado productor, aunque tampoco se excluye esta opción. En este concepto del papel del Estado se destaca la aplicación de políticas públicas orientadas a fortalecer la capacidad productiva en los sectores de mayor prioridad para el desarrollo, como es el caso, por ejemplo, de aquellos vinculados a la innovación tecnológica y productiva. Proponemos intervenciones inteligentes por parte del Estado, pero no contra el mercado, y evitar la exclusión dogmática del Estado como la que hicimos en años anteriores.

Tercero, se necesita un Estado que tenga la responsabilidad de disminuir las desigualdades sociales. El Estado cumple un papel indispensable en la defensa activa de la cohesión social y la lucha contra la pobreza. La eficiencia de los derechos políticos y civiles depende de un aparato estatal que garantice la observancia de la legalidad y condiciones materiales de vida dignas para la población, por la vía del reconocimiento y la cobertura de sus derechos económicos y sociales. Esto implica que el Estado asume dos funciones fundamentales: la de habilitador y la de compensador. Habilitador del ciudadano para que este acceda a una mayor igualdad de 
oportunidades a través de la educación, y compensador porque debe velar por el bienestar de los desamparados de la sociedad. Esto no significa asignar al Estado un papel protagónico en el crecimiento económico como vía para resolver los problemas de pobreza, lo que suplantaría las responsabilidades del sector privado en la esfera económica.

Cuarto, la experiencia moderna pone de relieve un nuevo relacionamiento del Estado con la empresa privada, del cual se han derivado importantes dividendos económicos y sociales. En ese campo cabe identificar nuevas modalidades de cooperación, como, por ejemplo, la coparticipación del Estado y la empresa privada en el financiamiento de la infraestructura, que sin duda será uno de los mayores retos para la economía regional en los próximos años.

Quinto, con relación al papel del Estado frente a la sociedad civil, se entiende hoy claramente que el fortalecimiento de la sociedad civil es algo estrechamente asociado a la reforma del Estado. Desde el BID sostuvimos repetidamente que no hay Estado capaz con una sociedad civil débil ni viceversa. Esto es, que no existe una sociedad civil fuerte sin el amparo de un Estado fuerte y eficiente. Sin embargo, no debe confundirse tamaño con fuerza ni grasa con músculo, como Prebisch lo señaló a menudo. El desarrollo requiere más Estado, más mercado y más sociedad civil, pero de manera coherente, que permita la convivencia y el refuerzo mutuo de las potencialidades máximas de cada una de las partes. Debe reforzarse creativamente esa relación, fomentando mecanismos de participación de la sociedad civil en las funciones del Estado. Esto es sin duda un gran desafío, que debe enfrentarse empezando por la eliminación de las sospechas recíprocas comunes en esta relación.

Sexto, el Estado tiene un papel fundamental en la orientación y formulación de las políticas de inserción internacional. Corresponde al Estado, en consulta y colaboración con el sector privado, adoptar las grandes decisiones que permitan desarrollar las relaciones de inserción internacional de los países. Esa tarea tiene importancia crítica en las actuales condiciones de creciente complejidad de las relaciones internacionales, tanto en el plano regional como a nivel mundial. El rol del Estado es de particular relevancia en el proceso de integración regional. Las solidaridades de hecho sobre las que se construye la integración económica y política dependen de un proceso de convergencia de intereses, valores y culturas, cuya columna vertebral depende de las capacidades institucionales de los países y, en particular, del papel del Estado. La integración es un proceso complejo y dinámico, que avanza mediante la superación de conflictos de adaptación de las estructuras económicas, políticas y sociales de los países. Todo ello difícilmente puede lograrse sin el liderazgo de las instituciones del Estado. Jean Monet solía decir que nada es posible sin las personas, pero que nada es duradero sin las instituciones. La integración regional, que en sí misma es un proceso de reforma del Estado, difícilmente puede avanzar sin la presencia de Estados capaces de enfrentar eficazmente los problemas de adaptación que ella trae consigo.

Séptimo, el Estado desempeña un papel de suma importancia en la innovación tecnológica. En las primeras etapas de la formación de las naciones, el Estado tuvo un rol fundamental en la educación del ciudadano. Hoy en día, la nueva frontera que se abre a la responsabilidad primaria del Estado es el apoyo al desarrollo de la tecnología y de la innovación tecnológica. Lo que ayer era la educación hoy es la extensión y la calidad de la educación. Lo de hoy es, además, la promoción de la investigación científica y la innovación tecnológica. Esto no desconoce el papel fundamental de la actividad privada en este campo, pero las distancias que nos separan del mundo desarrollado pueden aumentar si dejamos de hacer un esfuerzo masivo de desarrollo tecnológico, el cual requiere inevitablemente una acción efectiva del Estado.

Octavo, en el mundo cada vez más complejo en que vivimos es menester fortalecer la capacidad de análisis de las tendencias fundamentales de la economía, la sociedad y la política en el ámbito internacional. Vivimos en un mundo que avanza en todos los campos a ritmos sin precedente, dominados por las fuerzas de la globalización y el desarrollo vertiginoso de las tecnologías de la información y de las comunicaciones. Frente a ello debemos mantener, como condición de la más alta prioridad, la mayor capacidad de observación y seguimiento que nos sea posible. En esta tarea el Estado moderno debe promover un proceso permanente de reflexión por los sectores público y privado, que nos permita enfrentar los retos y aprovechar las oportunidades que nos plantean las distintas vertientes de la globalización.

Noveno, un componente institucional de probada idoneidad en el desarrollo de la capacidad de análisis de las grandes tendencias económicas, sociales y políticas en el ámbito nacional e internacional, lo constituyen las oficinas de planificación. Debemos corregir pasados abandonos de las tareas de análisis y de reflexión sobre el futuro. El pensamiento a largo plazo debe volver a ser un objetivo importante del Estado contemporáneo. No estamos abogando por el tipo de planificación 
centralizada, sino por la capacidad para proyectar tendencias a largo plazo sobre las cuales fundar las estrategias de desarrollo.

Décimo, otra función prioritaria del Estado moderno es la de promover los grandes consensos nacionales. Como sabemos, la concertación a distintos niveles entre Estado, empresa privada, sindicatos y sociedad civil ha sido una valiosa experiencia de muchos países desarrollados. Sin pretender diluir las responsabilidades propias de cada estamento, es útil apuntar a mecanismos que contribuyan a grandes consensos nacionales, que en las condiciones actuales de la región parecen tan útiles y necesarios. Considero que la formación y el fortalecimiento de los consejos económicos y sociales deberían ser merecedores de especial atención por parte del Estado.

Este verdadero decálogo de objetivos del nuevo Estado debería ser complementado con la definición o identificación de los requisitos e instrumentos idóneos y necesarios para lograrlos.

\section{Requisitos}

A mi juicio, la primera condición es la de contar con un sistema democrático robusto. Sin duda este es el requisito más importante y quizás su mención resulte algo novedosa. Talvez sea novedoso ligar el sostenimiento y profundización de la democracia con las capacidades del Estado. Pero es relativamente común vincular el mal funcionamiento de la democracia al desarrollo de su componente liberal, relacionando este con la debilidad de los mecanismos de limitación y división efectiva del poder que aseguran la protección de los derechos y libertades civiles y políticas. Sin embargo, se ha resaltado menos la debilidad causada por el déficit del llamado componente republicano de la democracia, según el cual el ejercicio de cargos públicos debe ser una actividad virtuosa, que supone una estricta sujeción a la ley y obediencia al interés público, sacrificando muchas veces el interés privado.

Una segunda condición es la de crear un servicio civil profesional, con una sólida posición institucional y sentido del deber, amparado por un marco normativo apropiado. La autonomía institucional del servicio civil, cubierto por estrictos criterios de igualdad, mérito y capacidad en el acceso y en el ordenamiento de la carrera, funciona en las democracias avanzadas como un contrapeso a la discrecionalidad política y del gobierno y como un freno a la arbitrariedad, salvaguardando los valores de la legalidad sin los cuales sería difícil la protección efectiva de los derechos y libertades de los ciudadanos.
La democracia no se consolidará sin una reforma del Estado que apueste por la instituciolización de un servicio civil profesional. El progreso de la democracia política, económica y social en los países desarrollados no se puede entender sin reconocer la fortaleza de sus instituciones administrativas. Como tampoco cabe extrañarse de que los países de la región con los mejores indicadores de cohesión social sean aquellos de mayor tradición institucional pública, inclusive con un servicio civil de carrera. Por tanto, se debe superar la politización de la administración pública y evitar su captura por intereses particulares que llevan al clientelismo y la influencia de las amistades. Este es un paso fundamental en el camino hacia el Estado que la democracia de América Latina necesita hoy en día.

Existe, asimismo, la necesidad de aumentar y mejorar la capacidad de gestión del gasto público. Un crecimiento sostenible y equitativo depende también de la calidad y la eficiencia de las políticas y de la gestión pública. Es indispensable entonces elevar la capacidad fiscal de los gobiernos, a la vez que su responsabilidad. En particular, es prioritario adaptar los sistemas de asignación de recursos para que respondan a las necesidades de los más pobres, y acomodar los sistemas de prestación a sus circunstancias específicas, permitiéndoles la participación y el compromiso directo. Para lograr todo ello, es imprescindible superar la politización de la administración pública y su captura por intereses particulares, como ya se señaló. La administración pública está llamada a constituir la plataforma institucional básica para el diseño e implementación de políticas públicas que respondan a los intereses generales de la sociedad.

Finalmente, cualquier planteamiento relativo a la reforma del Estado tendrá que superar tanto las hipotecas ideológicas relacionadas con ella, como la lógica que las reduce a simples problemas técnicos. La reforma del Estado es realizable solo a través de ajustes incrementales, que sean concebidos desde la economía política de lo posible. La realidad demuestra que son las ideas y no las ideologías las que permiten avanzar paulatinamente en la solución de los problemas. Eso afecta también el diseño del Estado, en que no hay muchas opciones viables unidas a banderas de diverso color, sino espacios de cambios graduales, normalmente transversales, que requieren grandes consensos y apoyos de la sociedad en su conjunto

Del mismo modo, concebir la reforma del Estado como un problema exclusivamente técnico, separado de la política, significa desconocer una realidad que reaparece con el tiempo bajo formas insospechadas. Es cada vez más evidente que los países que han sido 
capaces de progresar en forma sostenible no son los que han subordinado la lógica política a criterios puramente técnicos. Solo cuando la racionalidad técnica y política ha encontrado su acomodo armónico, donde las reformas se han presentado y discutido con transparencia y sin miedo a sus costos políticos, donde se ha invertido de forma transversal en conocimiento local y apropiación colectiva de planes y proyectos, solo en esos casos constatamos avances, quizás más lentos, pero también más sostenibles y equitativos.
Es importante destacar que, más allá de algunos consensos básicos en los equilibrios a nivel macro, los países con mayor éxito presentan una combinación muy diversa de modelos institucionales y políticos de reforma. Pero a la vez coinciden en haber desarrollado procesos innovadores en la búsqueda de respuesta a sus problemas, que representan equilibrios peculiares entre la racionalidad política y la técnica. Esta es quizá la lección más importante del proceso de reformas en países como Chile y Brasil. 